\title{
Erratum to: Distribution and Some Biological Features of Ixodid Ticks (Parasitiformes, Ixodidae) in Kuznetsk-Salair Mountain Area (Kemerovo Province, Russia)
}

\author{
A. V. Kovalevskiy ${ }^{a}$, K. S. Zubko ${ }^{a}$, A. R. Efimova ${ }^{b}$, E. M. Luchnikova ${ }^{a}$, O. M. Drozdova ${ }^{c}$ \\ ${ }^{a}$ Kemerovo State University, Kemerovo, 650000 Russia \\ *e-mail: passer125@yandex.ru \\ ${ }^{b}$ Center of Hygiene and Epidemiology of Kemerovo Province, Kemerovo, 650002 Russia \\ cKemerovo State Medical University, Kemerovo, 650056 Russia \\ Received February 24, 2018
}

DOI: $10.1134 / \mathrm{S} 0013873819010184$

The figure on p. 1381 should be as follows:

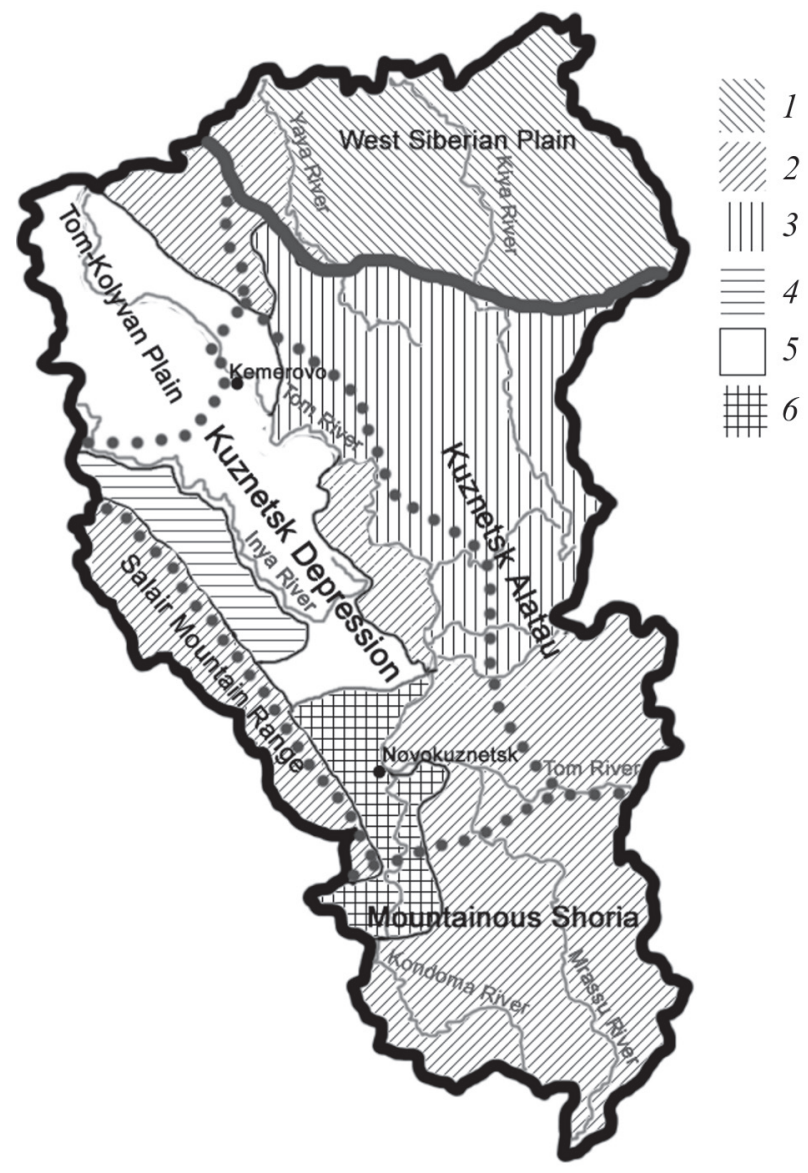

Schematic map of distribution of ixodid ticks attacking their hosts in pastures by physiographic regions of Kemerovo Province: 1 , the zone of distribution of I. persulcatus, I. pavlovskyi is present in small numbers in environs of some towns and villages; 2, the zone of joint dwelling of I. persulcatus and I. pavlovskyi; 3, the zone of distribution of I. persulcatus, I. pavlovskyi is unknown; 4, the zone where ticks of the genus Dermacentor prevail; 5 , the zone with simultaneous distribution of I. persulcatus, I. pavlovskyi, D. reticulatus, and D. silvarum; 6 , the zone of simultaneous distribution of I. persulcatus, I. pavlovskyi, D. reticulatus, D. silvarum, and Haemaphysalis concinna.

The original article can be found online at https://doi.org/ 10.1134/S0013873818090154. 